\title{
Patrones de personalidad asociados al comportamiento de consumo de alcohol en la ciudad de Cusco
}

\section{Personality patterns associated with alcohol consumption behavior in the city of Cusco}

Recibido: 25 de Julio 2018 | Aceptado: 26 de Setiembre 2018

\author{
Yency Acetuna $^{1} \&$ Franklin Soncco $^{1}$
}

\author{
${ }^{1}$ Universidad Nacional de San Antonio de Abad del Cusco \\ Correo electronico: yenchyacetuna@gmail.com
}

\section{Resumen}

La presente investigación relaciona la personalidad con el consumo de alcohol, siguiendo un diseño de corte transversal correlacional, la población está constituida por pacientes atendidos en los establecimientos de salud de la ciudad del Cusco con una muestra de 217 de 16 a 64 años entre varones y mujeres. El objetivo fue determinar la relación entre las variables, empleando los instrumentos: Inventario de Personalidad NEO-FFI y el Test de Identificación de los Trastornos Debidos al Consumo de Alcohol-AUDIT; el nivel significancia de Alpha de Cronbach $(0,61)$ para NEO-FFI y $(0,75)$ para AUDIT. De acuerdo a los resultados, se evidencia relaciones de dependencia entre las dimensiones de la personalidad neuroticismo, extraversión, apertura a la experiencia y conciencia; en cambio, no existe relación de dependencia entre la amabilidad y el consumo de alcohol. Por otra parte, se observan que las dimensiones neuroticismo, extraversión, apertura y conciencia se vinculan con el consumo de alcohol según las variables sociodemográficas las etapas de desarrollo adolescente, joven, adulto mayor; en ambos sexos y en procedentes del sector rural y urbano. En conclusión, Se evidencia relación entre las dimensiones de la personalidad neuroticismo, apertura a la experiencia, conciencia y el consumo de alcohol, y algunas relaciones significativas según las variables sociodemográficas.

Palabras clave: Dimensión de la personalidad, consumo de alcohol y pacientes.

\begin{abstract}
The present investigation relates the personality with the alcohol consumption, following a correlational cross-sectional design, the population is constituted by patients attended in the health establishments of the city of Cusco with a sample of 217 of 16 to 64 years between males and females. women. The objective was to determine the relationship between the variables, using instruments: Inventory of Personality NEO-FFI and Test of Identification of the Disorders Due to the
\end{abstract}


Consumption of Alcohol - AUDIT; the significant level of Cronbach's Alpha (0.612) for NEO-FFI and (0.759) for AUDIT. According to the results, dependence relations between the dimensions of personality neuroticism, extraversion, openness to experience and consciousness are evidenced; On the other hand, there is no dependency relationship between kindness and alcohol consumption. On the other hand, we observe that the neuroticism, extraversion, openness and awareness dimensions are linked to alcohol consumption according to the sociodemographic variables stages of adolescent, young and older adult development; in both sexes and from the rural and urban sectors. In conclusion, there is a relationship between personality dimensions, neuroticism, openness to experience, awareness and alcohol consumption, and some significant relationships according to sociodemographic variables.

Keywords: dimension of personality, alcohol consumption and patients

\section{Introducción}

La ciudad del Cusco, cuna de la cultura peruana, se ha caracterizado por presentar una mixtura de costumbres, ya que a lo largo de su historia ha sufrido una serie de cambios y transformaciones de manera progresiva de carácter sociocultural. El uso de bebidas alcohólicas es parte del desarrollo histórico de esta cultura.

La presente investigación surgió por la necesidad de conocer el problema del consumo de alcohol a través del conocimiento de la personalidad, ya que de acuerdo a Huillca (2008), la región del Cusco| presentó altos índices de consumo excesivo de alcohol, identificándose como la segunda región del sur del país con este problema, en ese mismo año se atendieron alrededor de mil pacientes en los diferentes establecimientos de salud los cuales también presentaron problemas a nivel personal, familiar y social.

Por tal motivo, se pretende obtener una información científica mejor contextualizada para poder abordar este tipo de problemas; de tal manera que se puedan desarrollar estrategias de atención más acorde a la necesidad de pacientes cusqueños, de forma complementaria, el estudio brinda conocimiento actualizado a todos los que están involucrados en el acompañamiento de estos pacientes, y sobre todo brinda estadísticas recientes sobre los índices de este problema.

El objetivo principal de este estudio es determinar la relación entre las dimensiones de la personalidad y consumo de alcohol y encontrar las mejores explicaciones a la dinámica de estas variables. Para los resultados se evaluaron a pacientes que recurren a los establecimientos de salud de la ciudad del Cusco; para desarrollar esta actividad se hizo uso de los instrumentos psicológicos (Inventario NEO-FFI y Test AUDIT).

En base a este estudio, se evidencia que existe relación entre algunas dimensiones de la personalidad y el consumo de alcohol, entre los más importantes encontramos: Las 
dimensiones de la personalidad neuroticismo $(\mathrm{N})$, extraversión $(\mathrm{E})$, apertura a la experiencia (O) y conciencia (C) vinculados al consumo de alcohol; en cuanto a las relaciones de estas variables de acuerdo a los datos sociodemográficos se evidencian algunas relaciones poco significativas que explican mejor en grupos específicos.

\section{Método}

El estudio responde a un enfoque cuantitativo por utilizar la recolección de datos para probar hipótesis en base a la medición y el análisis estadístico, optando por una investigación básica, para ampliar y profundizar conocimientos científicos existentes acerca de las dimensiones de personalidad y el consumo de alcohol (Carrasco, 2006). El alcance es de tipo descriptivo-correlacional; que describe, analiza e interpreta organizadamente un conjunto de fenómenos $\mathrm{y}$, correlacional identifica el grado $\mathrm{y}$ dirección de las variables. Con un diseño no experimental, ya que no hay manipulación ni asignación al azar de los participantes; transeccional para la recolección de datos en el periodo de tiempo sin hacer el seguimiento a los sujetos (Hernández, et al., 2014).

\section{Muestra}

Los participantes son 217 pacientes atendidos en los diferentes establecimientos de salud (Hospital Antonio Lorena, Hospital Regional, CLAS Centros de Salud de Wanchaq, Belenpampa, San Sebastián y San Jerónimo) de la ciudad del Cusco de enero a marzo 2017, de edades 16 a 64 años entre varones y mujeres. El tipo de muestreo no probabilístico intencional (Carrasco, 2006). Instrumentos y técnicas

\section{Instrumento.}

Inventario adaptado de los cinco rasgos de la personalidad (NEO-FFI).

\section{Confiabilidad y validez.}

El Inventario de Personalidad NEO-FFI, versión española y reducida del NEO PI de Costa y McCrae (2002), es un instrumento psicométrico del Modelo de los Cinco Factores que evalúa los cinco rasgos de personalidad sin tomar en cuenta las facetas de cada factor o suprarasgos. La versión española adaptada por TEA del Inventario de personalidad NEO-FFI presenta un nivel significativamente alto de consistencia interna, hallando grados de coeficiente Alfa de Cronbach entre .82 y .90; no obstante, la adaptación y validación del inventario de personalidad NEO-FFI, Cassaretto y Martínez (2011) demuestran favorablemente su valor psicométrico está por encima del .70; como consecuencia se asume que el NEO-FFI es un instrumento prometedor para evaluar de forma rápida y amplía la personalidad desde el modelo de los cinco factores.

En el Perú, Cassaretto y Martínez (2011), analizaron las propiedades psicométricas a través de dos estudios con estudiantes universitarios peruanos a quienes se les aplicó el instrumento obteniéndose una adecuada estructura factorial e índices de confiabilidad favorable para factores y/o rasgos de neuroticismo, 
extraversión, apertura, amabilidad y conciencia. De la misma forma también Cáceres (2016) en su investigación realizó el análisis de consistencia interna mediante el coeficiente Alpha de Cronbach donde obtuvo índices de confiabilidad aceptables para cuatro rasgos; ya que el rasgo de apertura a la experiencia no alcanzó el valor mínimo, fue neutralizado y se trabajaron con cuatro factores $(\mathrm{N}, \mathrm{E}$, A, C).

En el caso de esta investigación, a través del estadígrafo Alpha de Cronbach se obtuvo un índice de consistencia interna global de $\alpha=.61$ el cual indica que el instrumento NEO-FFI cumple con el requisito para ser considerada confiable (ver tabla 9). Este resultado es similar a otras investigaciones, considerándose que la prueba presenta un óptimo nivel de funcionamiento.

Para determinar la validez de constructo del inventario NEO-FFI, la estructura factorial de cada prueba se usó el índice de consistencia interna Alfa de Cronbach.

$$
\alpha=\frac{k}{k-1}\left(1-\frac{\Sigma s_{i}}{s_{t}}\right) \quad \Longrightarrow \alpha=\frac{n p}{1+p(n-1)}
$$

$\alpha=$ Alfa de Cronbach, $\mathrm{k}=$ Número de ítems, $\mathrm{Si}=$ Varianza de cada ítem, $\mathrm{St}=$ Varianza del total, $\mathrm{p}=$ Periodo de las correlaciones lineales de cada uno de los ítems, $\mathrm{n}=$ Muestra

Test de Identificación de los Trastornos Debidos al Consumo de Alcohol (AUDIT).

\section{Confiabilidad y validez.}

El AUDIT ha demostrado ser preciso en la detección de dependencia de alcohol en diversos países y entre los diversos criterios se sitúan como media en valores superiores de consistencia interna .80, (Babor, et al., 2001).

En el Perú, Duoglas Hospital Research Centre - McGill Unversity, Montreal Canadá en convenio con Instituto para la Paz y Desarrollo de Ayacucho - IPAZ Perú (2007) un Proyecto Estabilización Post Conflicto y Rehabilitación Psicosocial en Comunidades Alto Andinas del Perú, obtuvo un índice de consistencia interna favorable, similar a la versión original de la prueba.

Para esta investigación se procedió a obtener un índice de consistencia interna general Alfa de Cronbach de $\alpha=.75$ el cual indica que la prueba tiene el requisito para ser considerado confiable (ver tabla 10). Este resultado es similar a otras investigaciones, considerándose que la prueba presenta un óptimo nivel de funcionamiento.

Para determinar la validez de constructo del test AUDIT se usó el índice de consistencia interna Alfa de Cronbach. 


$$
\alpha=\frac{k}{k-1}\left(1-\frac{\sum s_{i}}{s_{t}}\right) \quad \Longrightarrow \alpha=\frac{n p}{1+p(n-1)}
$$

$\alpha=$ Alfa de Cronbach, $\mathrm{k}=$ Número de ítems, $\mathrm{Si}=$ Varianza de cada ítem, $\mathrm{St}=$ Varianza del total, $\mathrm{p}=$ Periodo de las correlaciones lineales de cada uno de los ítems, $\mathrm{n}=$ Muestra.

La técnica de recolección de datos para la estadística inferencial, se utilizó prueba de Chi Cuadrado para analizar la relación de ambas variables.

Para determinar el grado de relación entre las variables se utilizó Tau $b$ de Kendall, el cual permite conocer la magnitud y dirección de las relaciones existente entre las variables de una población (Wayne, 2002).

\section{Resultados}

Relación entre las cinco dimensiones de personalidad y consumo de alcohol

\section{Tabla 1}

Relación entre las dimensiones de personalidad y consumo de alcohol

\begin{tabular}{lcccc}
\hline \multicolumn{1}{c}{ Dimensiones } & Chi - Cuadrado & GL & $P$-ralor & Tau b de Kendall \\
\hline Neuroticismo & 29.28 & 4 & 0.000 & .23 \\
\hline Extraversión & 20.18 & 4 & 0.000 & -.14 \\
\hline Apertura a la experiencia & 19.55 & 4 & 0.001 & .21 \\
\hline Amabilidad & 4.27 & 4 & 0.371 & .02 \\
\hline Consciencia & 27.54 & 4 & 0.000 & -.19 \\
\hline
\end{tabular}

Nota: GL = Grados de Libertad. Adaptado: Elaboración de los autores.

Se observa que el resultado de la aplicación de la prueba Chi-Cuadrado de independencia al 95\% de confianza, es altamente significativo $(p=.000, \alpha<.05)$. Por lo que se determina, rechazar la hipótesis nula y aceptar la hipótesis alterna, esto indica que existe relación entre la dimensión neuroticismo y el consumo de alcohol. Y la prueba Tau $b$ de Kendall, se verifica relación directa entre las la dimensión neuroticismo y el consumo de alcohol (.23). Esto indica que los pacientes con altos niveles de neuroticismo se relacionan con un consumo de alcohol problemático.

Se aprecia que el resultado de la aplicación de la prueba Chi-Cuadrado de independencia al 95\% de confianza, es altamente significativo $(p=.000, \alpha<.05)$. Por consiguiente, rechazamos la hipótesis nula y aceptamos la hipótesis alterna; lo que significa que existe relación entre la dimensión de extraversión y consumo de alcohol. La aplicación de la prueba Tau $b$ de Kendall se observa una relación inversa entre la extraversión y el consumo de alcohol (-.14). Como consecuencia se infiere que un nivel alto de extraversión está relacionado a un consumo de alcohol bajo o no problemático. 
Se observa que el resultado de la aplicación de la prueba Chi-Cuadrado de independencia al 95\% de confianza, es altamente significativo ( $p=.001, \alpha<.05)$. Por lo tanto, se infiere que, hay razones suficientes para rechazar la hipótesis nula y aceptar la hipótesis alterna, lo cual significa que existe relación entre la dimensión apertura a la experiencia y consumos de alcohol. Y la aplicación del estadístico Tau $b$ de Kendall tiene relación directa entre la dimensión apertura a la experiencia y el consumo de alcohol (.21). Esto indica que los evaluados con altos niveles en la dimensión apertura a la experticia presentan a un consumo de alcohol alto $\mathrm{o}$ problemático.

Se evidencia que el resultado de la aplicación de la prueba Chi-Cuadrado de independencia al 95\% de confianza, con un bajo nivel de significación $(p=371, \alpha>05)$. En consecuencia, existe prueba suficiente para rechazar la hipótesis alterna y aceptar la hipótesis nula, lo que implica, que no existe relación entre la dimensión amabilidad y el consumo de alcohol. Según el resultado de aplicación del estadístico Tau $b$ de Kendall es que se puede inferir, relación inversa entre la dimensión amabilidad y el consumo de alcohol (.02). Esto indica que los evaluados con niveles altos en amabilidad se vinculan mejor a un consumo de alcohol no problemático.

En el análisis presentada, se aprecia el resultado de la aplicación de la prueba Chi-Cuadrado de independencia al 95\% de confianza, es altamente significativo $(p=.000$, $\alpha<.05)$. En consecuencia, existe razón suficiente para rechazar la hipótesis nula y aceptar la hipótesis alterna, lo cual denota que existe relación entre la dimensión conciencia y el consumo de alcohol. Según el resultado de aplicación del estadístico Tau $b$ de Kendall es que se puede inferir, relación inversa entre la dimensión conciencia y el consumo de alcohol (-.19). Esto indica que los evaluados con niveles altos en onciencia se vinculan mejor a un consumo de alcohol no problemático.

Dimensiones de la personalidad y consumo de alcohol según etapas del desarrollo 


\section{Tabla 2}

Relación entre las dimensiones de personalidad y consumo de alcohol según etapas del desarrollo

\begin{tabular}{|c|c|c|c|c|c|c|}
\hline \multirow{3}{*}{$\begin{array}{l}\text { Adolescencia } \\
\text { Personalidad }\end{array}$} & \multicolumn{5}{|c|}{ Estadísticos } & \multirow{3}{*}{$\begin{array}{l}\text { Tau b de } \\
\text { Kendall }\end{array}$} \\
\hline & \multicolumn{5}{|c|}{ Consumo de alcohol } & \\
\hline & $M$ & $D E$ & $\begin{array}{c}\text { Chi- } \\
\text { Cuadrado }\end{array}$ & GL & $P$-valor & \\
\hline Neuroticismo & 1.9 & 0.45 & 3.59 & 2 & 0.166 & .41 \\
\hline Extraversión & 2.5 & 0.51 & 3.33 & 1 & 0.068 & -.41 \\
\hline Apertura & 2.15 & 0.37 & 0.06 & 1 & 0.798 & -.06 \\
\hline Amabilidad & 2.2 & 0.52 & 1.61 & 2 & 0.448 & -.09 \\
\hline Conciencia & 2.5 & 0.51 & 3.33 & 1 & 0.068 & -.41 \\
\hline Juventud & $M$ & $D E$ & $\begin{array}{c}\text { Chi- } \\
\text { Cuadrado }\end{array}$ & GL & $P$-valor & $\begin{array}{l}\text { Tau b de } \\
\text { Kendall }\end{array}$ \\
\hline Neuroticismo & 1.87 & 0.51 & 30.73 & 4 & 0.000 & .27 \\
\hline Extraversión & 2.43 & 0.58 & 17.09 & 4 & 0.002 & -.17 \\
\hline Apertura & 2.04 & 0.33 & 23.63 & 4 & 0.000 & -.06 \\
\hline Amabilidad & 2.22 & 0.45 & 3.62 & 4 & 0.460 & -.06 \\
\hline Conciencia & 2.48 & 0.55 & 31.11 & 4 & 0.000 & -.18 \\
\hline Adultez & $M$ & $D E$ & $\begin{array}{c}\text { Chi- } \\
\text { Cuadrado }\end{array}$ & GL & $P$-valor & $\begin{array}{l}\text { Tau b de } \\
\text { Kendall }\end{array}$ \\
\hline Neuroticismo & 1.84 & 0.48 & 3.2 & 4 & 0.525 & .16 \\
\hline Extraversión & 2.52 & 0.50 & 0.16 & 2 & 0.925 & -.01 \\
\hline Apertura & 2.05 & 0.26 & 2.96 & 4 & 0.563 & -.01 \\
\hline Amabilidad & 2.34 & 0.47 & 2.15 & 2 & 0.341 & .09 \\
\hline Conciencia & 2.39 & 0.49 & 3.75 & 2 & 0.153 & -.18 \\
\hline Adulto mayor & $M$ & $D E$ & $\begin{array}{c}\text { Chi- } \\
\text { Cuadrado }\end{array}$ & GL & $P$-valor & $\begin{array}{l}\text { Tau b de } \\
\text { Kendall }\end{array}$ \\
\hline Neuroticismo & 2.00 & 0.00 & & & & \\
\hline Extraversión & 2.50 & 0.53 & 4.8 & 1 & 0.028 & -.77 \\
\hline Apertura & 2.25 & 0.46 & 0.17 & 1 & 0.673 & -.15 \\
\hline Amabilidad & 2.25 & 0.71 & 0.89 & 2 & 0.641 & -.06 \\
\hline Conciencia & 2.25 & 0.71 & 4.8 & 2 & 0.091 & .35 \\
\hline
\end{tabular}

Nota: $\mathrm{M}=$ media, $\mathrm{DE}=$ Desvíación Estándar, GL = Grados de Libertad. Adaptado: Elaboración de los autores.

De acuerdo a lo presentado en la tabla 2, la aplicación de la prueba Chi-Cuadrado de independencia al 95\% de confianza, con un nivel de significación baja para las cinco dimensiones y el consumo de alcohol ( $\alpha>05)$. Esto indica que no existe relación de dependencia entre las cinco dimensiones de la personalidad y el consumo de alcohol según la adolescencia. Como resultado de la prueba estadística Tau $b$ de Kendall se observan relaciones moderados e inversas en las dimensiones extraversión (-.41) y conciencia (-.41), y una relación moderada y directa 
en la dimensión neuroticismo (.41). Lo cual indica que los adolescentes con altos niveles de extraversión y conciencia presentan un consumo noproblemático, por otra parte, los que presentan altos niveles de neuroticismo presentan un consumo de alcohol elevado o problemático.

Se observa que el resultado de la aplicación de la prueba Chi-Cuadrado de independencia al 95\% de confianza, es altamente significativo para las dimensiones neuroticismo $(p=.000, \alpha<.05)$, extraversión $(p=.002, \alpha<.05)$, apertura $(p=.000, \alpha<.05)$ y conciencia $(p=.000, \alpha<.05)$. Lo cual denota que existe relación de dependencia entre las dimensiones de la personalidad neuroticismo, extraversión, apertura a la experiencia y conciencia con el consumo de alcohol según la juventud. Según los resultados de la prueba Tau $b$ de Kendall, se evidencia relaciones poco significativas entre las dimensiones de la personalidad y el consumo de alcohol, se aprecian relaciones inversas en las dimensiones extraversión (-.17) y conciencia (-.18); y una relación directa en la dimensión neuroticismo (.27). Esto significa que los jóvenes con altos niveles de extraversión y conciencia presentan un consumo de alcohol no problemático, en cambio, los que presentan niveles altos de neuroticismo tienen un consumo de alcohol elevado o problemático.

El resultado se aprecia de la aplicación de la prueba Chi-Cuadrado de independencia al 95\% de confianza, un nivel de significación baja para cada dimensión ( $\alpha>05)$. Por lo que se tiene razón suficiente para aceptar la existencia de relación de dependencia entre las dimensiones de las cinco dimensiones de la personalidad y el consumo de alcohol según la adultez. En base a los resultados de la prueba Tau $b$ de Kendall, se observa una relación baja e inversa entre la dimensión conciencia y el consumo de alcohol (-.77). Esto indica que los adultos con predominancia en la dimensión conciencia presenta un consumo de alcohol no problemático.

Se observa que el resultado de la aplicación de la prueba Chi-Cuadrado de independencia al 95\% de confianza, un nivel de significación alta para la dimensión extraversión $(p=.028, \alpha<.05)$. Por lo que, se acepta la existencia de relación de dependencia entre la extraversión y el consumo de alcohol según la adultez mayor. Según la prueba Tau $b$ de Kendall, se observa una relación alta e inversa entre la dimensión extraversión y el consumo de alcohol (-.77), en cambio, la dimensión conciencia se relaciona directamente al consumo de alcohol (.35). Esto indica que los adultos mayores con predominancia en las dimensiones extraversión presentan un consumo de alcohol no problemático, en cambio, los que presentan niveles altos de conciencia tienen un consumo alto o problemático de alcohol. 


\section{Dimensiones de la personalidad y consumo de alcohol según sexo}

\section{Tabla 3}

Relación entre las dimensiones de personalidad y consumo de alcohol según el sexo

\begin{tabular}{|c|c|c|c|c|c|c|}
\hline \multirow[b]{3}{*}{ Masculino } & \multicolumn{5}{|c|}{ Estadisticos } & \\
\hline & \multicolumn{6}{|c|}{ Consumo de alcohol } \\
\hline & $M$ & $D E$ & $\begin{array}{c}\text { Chi- } \\
\text { Cuadrado } \\
\end{array}$ & GL & $P$-valor & $\begin{array}{l}\text { Tau b de } \\
\text { Kendall }\end{array}$ \\
\hline Neuroticismo & 1.84 & 0.44 & 25.74 & 4 & 0.000 & .24 \\
\hline Extraversión & 2.53 & 0.51 & 2.94 & 4 & 0.568 & -.11 \\
\hline Apertura & 2.09 & 0.31 & 3.19 & 4 & 0.525 & .00 \\
\hline Amabilidad & 2.28 & 0.47 & 2.75 & 4 & 0.601 & .03 \\
\hline Conciencia & 2.48 & 0.50 & 5.89 & 2 & 0.050 & -.18 \\
\hline Femenino & $M$ & $D E$ & $\begin{array}{c}\text { Chi- } \\
\text { Cuadrado }\end{array}$ & GL & $P$-valor & $\begin{array}{l}\text { Tau b de } \\
\text { Kendall }\end{array}$ \\
\hline Neuroticismo & 1.95 & 0.58 & 6.64 & 4 & 0.156 & .20 \\
\hline Extraversión & 2.32 & 0.60 & 19.98 & 4 & 0.002 & -.24 \\
\hline Apertura & 1.96 & 0.32 & 16.20 & 4 & 0.003 & -.23 \\
\hline Amabilidad & 2.21 & 0.49 & 3.49 & 4 & 0.479 & -.02 \\
\hline Conciencia & 2.33 & 0.60 & 16.47 & 4 & 0.002 & -.22 \\
\hline
\end{tabular}

Nota: $\mathrm{M}=$ media, $\mathrm{DE}=$ Desviación Estándar, $\mathrm{GL}=$ Grados de Libertad. Adaptado: Elaboración de los autores.

El resultado de la aplicación de la prueba Chi-Cuadrado de independencia al $95 \%$ de confianza, es altamente significativo para las dimensiones neuroticismo $(p=.000, \alpha<.05)$ y conciencia $(p=050, \alpha<.05)$. Por lo que se acepta las relaciones de dependencia entre las dimensiones de la personalidad neuroticismo y conciencia con el consumo de alcohol según el sexo masculino. De acuerdo a la aplicación del estadístico Tau b de Kendall se observa una relación moderada directa en la dimensión de personalidad neuroticismo (.24) e inversa en la dimensión conciencia (-.18). Lo cual significa que los evaluados de sexo masculino con predominancia en la dimensión neuroticismo presentan un consumo elevado o problemático, por otra parte, los que tienen predominancia en la dimensión conciencia presentan un consumo no problemático de alcohol.

La prueba Chi-Cuadrado de independencia al 95\% de confianza, es altamente significativo para las dimensiones extraversión $(p=.002, \alpha<.05)$, apertura a la experiencia $(p=.003, \alpha<.05)$ y conciencia $(p=.002, \alpha<.05)$. Con tales resultados se acepta relaciones de dependencia entre las dimensiones de la personalidad extraversión, apertura y conciencia con el consumo de alcohol según el sexo femenino. Los resultados de la aplicación de la prueba Tau $b$ de Kendall según el sexo femenino evidencia relaciones moderadas e inversas en las dimensiones extraversión (-.24), apertura a la experiencia (-.23) y conciencia (-.22); en tanto, la dimensión de personalidad 
neuroticismo está relacionado directamente (.20). Por tal razón se demuestra que las mujeres con altos niveles de extraversión, apertura y conciencia presentan un consumo de alcohol no problemático, en contraste, las que presentan neuroticismo alto tienen un consumo de alcohol problemático.

\section{Dimensiones de la personalidad y consumo de alcohol según procedencia}

Tabla 4

Relación entre las dimensiones de personalidad y consumo de alcohol según procedencia

\begin{tabular}{|c|c|c|c|c|c|c|}
\hline \multirow[b]{3}{*}{ Rural } & \multicolumn{5}{|c|}{ Estadisticos } & \\
\hline & \multicolumn{6}{|c|}{ Consumo de alcohol } \\
\hline & $M$ & $D E$ & $\begin{array}{c}\text { Chi- } \\
\text { Cuadrado }\end{array}$ & GL & P-valor & $\begin{array}{l}\text { Tau b de } \\
\text { Kendall }\end{array}$ \\
\hline Neuroticismo & 2.00 & 0.00 & & & & \\
\hline Extraversión & 2.44 & 0.52 & 1.87 & 4 & 0.759 & -.11 \\
\hline Apertura & 2.09 & 0.28 & 0.99 & 2 & 0.608 & -.01 \\
\hline Amabilidad & 2.17 & 0.46 & 4.86 & 4 & 0.301 & .08 \\
\hline Conciencia & 2.35 & 0.50 & 15.28 & 4 & 0.004 & -.37 \\
\hline Urbano marginal & $M$ & $D E$ & $\begin{array}{c}\text { Chi- } \\
\text { Cuadrado }\end{array}$ & GL & $P$-valor & $\begin{array}{l}\text { Tau b de } \\
\text { Kendall }\end{array}$ \\
\hline Neuroticismo & 1.41 & 0.49 & 3.15 & 2 & 0.204 & .10 \\
\hline Extraversión & 2.54 & 0.50 & 2.75 & 2 & 0.253 & .01 \\
\hline Apertura & 2.11 & 0.36 & 2.53 & 4 & 0.638 & .13 \\
\hline Amabilidad & 2.39 & 0.49 & 0.21 & 2 & 0.899 & .05 \\
\hline Conciencia & 2.65 & 0.48 & 0.20 & 2 & 0.903 & -.02 \\
\hline Urbano & $M$ & $D E$ & $\begin{array}{c}\text { Chi- } \\
\text { Cuadrado }\end{array}$ & GL & $P$-valor & $\begin{array}{l}\text { Tau b de } \\
\text { Kendall }\end{array}$ \\
\hline Neuroticismo & 2.19 & 0.39 & 14.73 & 2 & 0.001 & .40 \\
\hline Extraversión & 2.46 & 0.60 & 18.80 & 4 & 0.001 & -.26 \\
\hline Apertura & 1.97 & 0.29 & 15.97 & 4 & 0.003 & -.28 \\
\hline Amabilidad & 2.24 & 0.46 & 1.58 & 4 & 0.811 & -.00 \\
\hline Conciencia & 2.32 & 0.55 & 20.09 & 4 & 0.000 & -.65 \\
\hline
\end{tabular}

Nota: $\mathrm{M}=$ media, $\mathrm{DE}=$ Desviación Estándar, $\mathrm{GL}=$ Grados de Libertad. Adaptado: Elaboración de los autores.

Según lo presentado en la tabla 4, se contempla que el resultado de la aplicación de la prueba Chi-Cuadrado de independencia al 95\% de confianza, un nivel de significación alta para la dimensión conciencia $(p=.004, \alpha<.05)$. Por tal razón se acepta la existencia de relación de dependencia entre la dimensión de la personalidad conciencia y el consumo de alcohol según la procedencia rural. Según la aplicación de la prueba Tau b de Kendall, se observa una relación moderada e inversa entre la dimensión conciencia y el consumo de alcohol (-.37). Como consecuencia se infiere que los pacientes de procedencia rural con altas puntuaciones en la dimensión conciencian presentan un consumo de alcohol 
no problemático.

La prueba Chi-Cuadrado de independencia al 95\% de confianza, un nivel de significación baja para las cinco dimensiones $(\alpha>05)$. Por tanto, no existe relación de dependencia entre las dimensiones de la personalidad y el consumo de alcohol según la procedencia urbano marginal.

El resultado de la aplicación de la prueba Chi-Cuadrado de independencia al 95\% de confianza, altamente significativa para las dimensiones neuroticismo $(p=.001$, $\alpha<.05)$, extraversión $(p=.001, \alpha<.05)$, apertura $(p=.003, \alpha<.05)$ y conciencia $(p=.000$, $\alpha<.05)$. Por tanto, existe relación de dependencia entre las dimensiones de la personalidad neuroticismo, extraversión, apertura y conciencia con el consumo de alcohol según la procedencia urbana.

Por otro lado, la aplicación de la prueba Tau b de Kendall, se infiere que existe relaciones inversas entre la extraversión (-.26), apertura $(-0.27)$ y conciencia $(-.65)$, y una relación directa entre la dimensión neuroticismo y el consumo de alcohol (.40). Lo que significa que, los pacientes procedentes de zonas urbanas con altos noveles en extraversión, apertura y conciencia se relacionan con un consumo no problemático, por otro lado, los que tienen mayores nivele en la dimensión neuroticismo se relacionan con un consumo de alcohol dependiente.

\section{Discusión}

El resultado obtenido mediante la prueba no paramétrica de independencia $C h i$ Cuadrado y Tau b de Kendall, evidencia que existe una relación significativa y directa entre la dimensión neuroticismo y el consumo de alcohol; por tanto, las personas que presentan consumo problemático de alcohol se caracterizan por ser propensos de presentar ideas irracionales, dificultades para controlar sus impulsos y déficits para enfrentar situaciones estresantes, propios del neuroticismo (Costa y McCrae, 2012). Este resultado guarda relación con lo sostenido por Cáceres (2016), Aguirre (2016) y Belloch, et al., (2008) quienes también refieren que existe una determinada personalidad en sujetos que consumen alcohol caracterizado por conductas agresivas, violentas y poca capacidad de afrontar el estrés, características propias del neuroticismo. Mestre, et al., (2005) indica que el neuroticismo y la frecuencia cantidad del consumo de alcohol tiene una correlación directa significativa. Adicionalmente, estudios en estudiantes de bachillerato y universitarios de Gil (2012) y Pilatti, et al., (2014) muestran la importancia del neuroticismo para explicar el consumo de alcohol en dicha población, donde se evidencia que el consumidor problemático es inestabilidad emocional, presenta bajo nivel de autocontrol, sobre todo una elevada impulsividad $\mathrm{y}$ agresividad; estas características se presentan principalmente en sujetos adolescentes y jóvenes con neuroticismo alto. Los resultados de la investigación son fortalecidos con estudios efectuados en el contexto internacional y nacional.

De la misma forma, la dimensión de personalidad que se vincula inversamente mejor con el consumo de alcohol es la extraversión; por ende, los evaluados que presentan un consumo de alcohol no problemático se caracterizan 
principalmente por tener comportamientos gregarios, ser locuaces, alegres, divertidos, ser apasionados por las actividades que realizan y son enérgicos (Costa y McCrae, 2002). Lo cual resulta congruente con lo reportado en investigaciones anteriores a nivel nacional, Arana (2013) y Cáceres (2016) encontraron relaciones entre la extraversión y el consumo de alcohol problemático, episodios de exceso y cantidad como estrategia de afrontamiento. Siguiendo un diseño distinto, Aguirre (2016) describió el uso de habilidades y autoexpresiones sociales en sujetos que consumen bebidas alcohólicas. De forma similar, Gil (2012) siguiendo el mismo método de estudio señala que existe relación entre el rasgo introversión o extraversión baja y el consumo de alcohol.

Asimismo, se alega relación significativa y directa entre la dimensión apertura a la experiencia y el consumo de alcohol, por tanto, se infiere que las personas que presentan consumo problemático de alcohol se caracterizan por poseer comportamientos poco convencionales, ligados a la creatividad u originalidad, así como, preferencias a lo novedoso, ser osados y atrevidos. Estos resultados son similares a los hallazgos de Castañeda y Chaves (2013), Pilatti, et al., (2014) y Arana (2013) quienes señalan la existencia de relación significativa entre el consumo de alcohol y el estilo de personalidad innovación. Por otra parte, Gil (2012) evidencia relación inversamente significativa entre la dimensión apertura a la experiencia y el consumo de alcohol, esto nos da a conocer que la apertura a la experiencia baja está relacionada a bebedores $\mathrm{u}$ consumidores de alcohol. Los estudios anteriores en cuanto a la existencia de relación, afirman lo encontrado con esta investigación.

De la misma forma, se demuestra que existe relación inversa y significativa entre la dimensión conciencia y el consumo de alcohol, los pacientes que presentan consumo de alcohol problemático se caracterizan por ser menos rigurosos en aplicar principios morales y descuidados en luchar por sus objetivos, así también, desorganizados, impuntuales, impulsivos, poco comprometidas en cuestiones académicas y nada solidarios (Costa y McCrae, 2002). Este resultado es ratificado por el hallazgo reportados en la investigación realizada por Gil (2012) quien indica que los adolescentes que no beben o beben ocasionalmente tienen una capacidad de autocontrol efectiva y por el contrario aquellos sujetos con puntuaciones bajas en conciencia son bebedores activos ya sean perjudiciales o dependientes, esto se explica por las capacidades de regulación y control de nuestro cerebro, ya que no están muy bien desarrolladas en los adolescentes y la población de este estudio se enfoca en las diferentes etapas de desarrollo (adolescentes, jóvenes, adultos y adultos mayores). Sin embargo, los hallazgos en el estudio de Arana (2013) no evidencian relación entre estas variables (rasgo de personalidad conciencia y consumo de alcohol), esta diferencia también podría obedecer a causas culturales.

Por otro lado, la dimensión amabilidad describe tendencias interpersonales, según Costa y McCrae (2002), el hallazgo evidencia que la dimensión amabilidad no guardan relación con el consumo de alcohol, lo cual es semejante a los hallazgos de Arana (2013), quien hizo su estudio en una población de estudiantes universitario, no evidencia relación entre el rasgo de personalidad amabilidad y el alto o bajo consumo de alcohol. Por otra parte, Mestre, et al., (2005), Gil (2012) y Pilatti, et al., (2014) quienes encontraron relaciones fuertes en esta dimensión, de esta forma las personas con 
amabilidad baja se describen como más propensos a consumir altos niveles de bebidas alcohólicas.

Al relacionar las dimensiones de la personalidad y el consumo de alcohol en función a las etapas del desarrollo, se infiere relaciones significativas en algunas etapas del desarrollo (adolescencia, juventud, adultez y adultez mayor). Los adolescentes evidencian relaciones significativas en las dimensiones neuroticismo, extraversión y conciencia con el consumo de alcohol, esto indica que los adolescentes con neuroticismo alto presentan un consumo problemático de alcohol; por otro lado, los adolescentes con extraversión y conciencia alta presentan un consumo de alcohol no problemático. En cambio, el grupo de los jóvenes tiene relaciones significativas en las dimensiones neuroticismo, extraversión, apertura a la experiencia y conciencia, esto nos demuestra que los niveles altos de extraversión, apertura a la experiencia y conciencia presentan un consumo de alcohol no problemático, en cambio, los jóvenes con neuroticismo alto presentan un consumo de alcohol problemático. No se evidencian relaciones significativas en el grupo de los adultos, pero en el grupo de los adultos mayores, se observa niveles altos de extraversión que presentan un consumo de alcohol no problemático. Estos resultados son semejantes a los estudios descritos por Gil (2012), quien describe el perfil de personalidad en estudiantes bebedores en función a los cinco rasgos, como poco organizadas, poco cultas, poco responsables e irrespetuosos, por otra parte, Arana (2013) también señala que los jóvenes extrovertidos por lo general tienden a desarrollar un consumo problemático. De acuerdo a los resultados evidenciados, los adultos y adultos mayores con bajas puntuaciones en conciencia no presentan problemas con el consumo de alcohol, tienen dificultades a controlar sus impulsos, participan activa y asertivamente en grupos sociales, lo cual también encuentra parecido con otros estudios. En contraste, los adultos y adultos mayores que no presenta problemas al consumir bebidas alcohólicas son por lo general reflexivas, sensatas y prudentes comparado con las personas que tienen un consumo problemático que procuran satisfacer necesidades sociales y de pertenencia, por tanto, mantienen relaciones íntimas y afectuosas con otras personas significativas de su entorno (Castañeda y Chaves, 2013).

Los resultados de este estudio según la variable sociodemográfica sexo evidencia relaciones altamente significativas en las dimensiones neuroticismo y conciencia con el consumo de alcohol en pacientes de sexo masculino, por otro lado, las dimensiones extraversión, apertura a la experiencia y conciencia están relacionados significativamente con el consumo de alcohol en pacientes de sexo femenino. Los evaluados de sexo masculino con niveles altos en neuroticismo presentan un consumo de alcohol elevado o problemático, por otro lado, los que presentan conciencia alto tienen un consumo no problemático. En cambio, las evaluadas de sexo femenino con niveles altos de extraversión, apertura a la experiencia y conciencia presentan un consumo de alcohol no problemático. Este resultado es apoyado con los estudios de (Castañeda y Chaves, 2013) quienes demuestran la existencia de relaciones significativas para hombres entre el tipo de consumo de alcohol y el estilo de personalidad, se comprueba que la innovación está relacionada con el rasgo de apertura a la experiencia, la increpación está relacionada a la responsabilidad y el dominio que es explicado al igual que la extraversión, debido a que poseen relaciones parecidas al inventario NEO-FFI. Entre tanto las mujeres tuvieron mejores relaciones entre el 
consumo de alcohol y el estilo de personalidad modificación que se explica con la dimensión extraversión, la adecuación explicada con la conciencia y tienen pocas habilidades para afrontar el estrés. Por otra parte, se evidencia que gran proporción de estudiantes varones que consumen alcohol presentan un nivel alto de habilidades sociales.

Las relaciones en función a la procedencia en este estudio demuestran que la dimensión conciencia alta se relacionan mejor con el consumo de alcohol no problemático en procedentes del sector rural, lo cual denota que las personas procedentes de este sector por lo general son más responsables, organizados, cuidadosos y ambiciosos prefieren no consumir alcohol o al hacerlo, lo hacen moderadamente. Por otra parte, se infiere que las dimensiones extraversión, apertura a la experiencia y conciencia alta esta mejor vinculado a un consumo de alcohol no problemático; en cambio, los que presentan alto neuroticismo presentan un consumo problemático en procedentes del sector urbano, lo cual indica que los pacientes responsables, sociables, abiertas a nuevas experiencias y costumbres se vinculan a un consumo de alcohol no problemático, comparado con pacientes que son inestables afectivamente y por lo general tienen problemas al afrontar le estrés presentan un consumo de alcohol problemático. Este resultado muestra estadísticas parecidas al de otras investigaciones; se sabe que la modalidad predominante de consumo de alcohol en los sectores rurales es moderada en casi el $50 \%$ en comparación al alcoholismo, la predominancia de consumo se sitúa en el sexo masculino (Ramírez y Olivera, 2003). Los sujetos que presentan una intoxicación de alcohol o un consumo de alcohol problemático generalmente son las personas de procedencia del sector urbano (Paucar, 2010). No obstante, los criterios tomados para determinar la relación en cuanto a la procedencia es propuesta propia de los autores, puesto que la ciudad del Cusco conglomera a sujetos que viven a muy poca distancia entre procedentes del sector rural, urbano y urbano marginal, por tanto, las influencias hacen que puedan compartir muchas características en común, también se considera que la procedencia para ser considerada como tal, tiene muchas otras características específicas.

\section{Conclusiones}

El consumo de alcohol problemático se presenta en pacientes con niveles altos en neuroticismo y apertura a la experiencia, por otro lado, un consumo de alcohol no problemático se vincula a pacientes con niveles altos en extraversión y conciencia.

Se conoce algunas relaciones entre las dimensiones de la personalidad y el consumo de alcohol según la variable sociodemográfica etapas de vida, primero, las dimensiones de la personalidad neuroticismo, extraversión y conciencia se relacionan significativamente con el consumo de alcohol en adolescentes; segundo, las dimensiones neuroticismo, extraversión, apertura a la experiencia y conciencia establecen relaciones significativas con el consumo de alcohol en jóvenes; tercero, solo la dimensión extraversión se relaciona significativa con el consumo de alcohol en adultos mayores.

Se determina la existencia de relación entre algunas dimensiones de la 
personalidad y el consumo de alcohol según la variable sociodemográfica sexo, las dimensiones neuroticismo y conciencia se relacionan significativamente con el consumo de alcohol en los pacientes de sexo masculino; en cambio, las dimensiones extraversión, apertura a la experiencia y conciencia establecen relaciones significativas con el consumo de alcohol en los pacientes de sexo femenino.

Finalmente, la relaciones entre algunas dimensiones de la personalidad y el consumo de alcohol según la variable sociodemográfica procedencia, la dimensión de la personalidad neuroticismo se relaciona significativamente con el consumo de alcohol en pacientes de procedencia rural; en cambio, las dimensiones neuroticismo, extraversión, apertura a la experiencia y conciencia se relacionan significativamente con el consumo de alcohol en pacientes de procedencia urbano.

\section{Declaración de conflicto de interés}

Los autores declaran no tener ningún tipo de conflicto de intereses 


\section{Referencias}

Aguirre, M. (2016). Habilidades sociales en estudiantes del primer ciclo que consumen y no consumen alcohol. (Tesis para optar al título de Psicóloga). Universidad Andina del Cusco. Perú.

Allport, G. (1971). ¿Qué es la personalidad? Buenos Aires, Argentina: Ediciones Siglo Veinte.

Allport, G. (1974). Psicología de la personalidad. Buenos Aires, Argentina: Paidós.

Arana, M. y Pérez, C. (2013). Factores de personalidad en estudiantes universitarios según características del consumo de alcohol. (Tesis para optar al título de Licenciada en Psicología Clínica). Pontificia Universidad Católica del Perú. Perú

Asociación Americana de Psiquiatría. (2014). Manual diagnóstico y estadístico de los trastornos mentales - DSM 5 (5ta ed.). Madrid, España: Editorial Médica Panamericana.

Ávila, M. (2012). Factores personales y psicosociales de los adolescentes en el consumo de alcohol. (Tesis doctoral). Universidad de Alicante. España.

Babor, T., Higgins, J., y Monteiro, M. (2001). AUDIT: Cuestionario de Identificación de los Trastornos Debidos al Consumo de Alcohol. Pautas Para su Utilización en Atención Primaria. Ginebra: Organización Mundial de la Salud, Generalitat Valenciana.

Belloch, A., Sandín, B. y Ramos, F. (2008). Manual de psicopatología (Vol. 1). Madrid, España: McGraw Hill.

Brown, T., Errázuriz, C., Pedersen, D., Gamarra, J., Huayllasco, E. y Berrocal, J. (2007). Cuestionario de Identificación de los Trastornos debido al Consumo de Alcohol AUDIT. Proyecto Estabilización Post Conflicto y Rehabilitación Psicosocial en Comunidades Alto Andinas del Perú. Versión Español/quechua.

Cáceres, E. (2016). Factores de personalidad y estrategias de afrontamiento en jóvenes consumidores de marihuana. (Tesis para optar al título de Licenciada en Psicología Clínica). Pontificia Universidad Católica del Perú. Perú.

Carrasco, S. (2006). Metodología de la investigación científica. (1 ra reimpresión). Lima, Perú: San Marcos.

Carver, Ch. y Scheier, M. (1997). Teorías de la Personalidad(3ra ed.). México, D.L.: Pearson Educación.

Carver, Ch. y Scheier, M. (2014). Teoría de la Personalidad(7ma ed.). México, D.L.: Pearson Educación. 
Cassaretto, M. (2009). Relación entre las cinco grandes dimensiones de la personalidad y el afrontamiento en estudiantes preuniversitarios de Lima Metropolitana. (Tesis de maestría). Universidad Nacional Mayor de San Marcos. Perú.

Castañeda, J. y Chaves, L. (enero-junio 2013). Relaciones entre estilos de personalidad y tipos de consumo de alcohol. Cali, Colombia. Pontificia Universidad Javeriana. Revista Pensamiento Psicológico. Vol. 1, núm. 1, p. 55-69.

Cervone, D. y Pervin, L. (2009). Personalidad Teoría e Investigación (2da ed.). México, DL.: El Manual Moderno.

Cloninger, S. (2003). Teorías de la personalidad(3ra ed.). México: Pearson Educación.

Collazos, J. (2010). Perfil Epidemiológico de los Accidentes de Tránsito en el Perú, 2005-2009. Revista Perú Med Exp Salud Pública. Vol. 27, núm. 2, p. 162-169.

Costa, P. y McCrae, R. (1992). Inventario de Personalidad NEO Revisado (NEO PI-R) y el Inventario de los Cinco Factores NEO (NEO-FFI) Professional Manual Odessa. Florida, EEUU: Psychological Assessment Resources.

Costa, P. y McCrae, R. (2008). Inventario de Personalidad NEO Revisado (NEO PI-R) e Inventario NEO reducido de cinco factores (NEO-FFI) Manual profesional. Madrid, España: TEA Ediciones.

Cruz, J. y Rojas, R. (24 de diciembre del 2016). Consumo de alcohol aumenta entre las adolescentes peruanas. Diario Perú21, p. 01.

Decreto Supremo No 012-2009-SA. Ley que regula la comercialización, consumo y publicidad de bebidas. Lima, Perú. 2009. Art. 14, p. 1-7.

Del Rio, M.C. (2002). Alcohol, jóvenes y accidentes de tráfico. Universidad de Valladolid. España. Trastornos adictivos: Órgano Oficial de la Sociedad española de la Taxonomía. Vol. 4, núm. 1, p. 20-27.

Dirección Regional de Salud Cusco (2013). Documento de Investigación Operativa de Análisis de Situación de la Salud en la Región de Cusco, al mes de diciembre del año 2012. (1ra ed.) Cusco, Perú: Dirección Regional de Salud Cusco.

Eysenck, H. (1990). Contribución genética ambiental a las diferencias individuales. Tres mayores dimensiones de la personalidad. Revista de personalidad. núm. 58, p. 245-261.

Eysenck, H. y Eysenck, M. (1987). Personalidad y diferencias individuales (ed. española) Madrid, España: Pirámide.

García, M., Borasino, P. y Amaya, E. (agosto del 2011) Conductas sexuales de riesgo post consumo de alcohol y/o drogas ilegales en estudiantes de carreras no médicas de la universidad los Ángeles de Chimbote, centro académico Sullana, 
durante diciembre 2008-2009. Perú. DEVIDA. Revista peruana de drogodependencias. Análisis e investigación. Vol. VII, núm. 1.

Gil, S. (2012). Alcoholismo de Fin de Semana en Adolescentes: Funcionamiento Prefrontal, Rasgos Psicopatológicos y de Personalidad. (Tesis doctoral). Universidad Nacional Complutense de Madrid. España.

Guardia, J., Jiménez, M., Pascual, P., Flores, G. y Contel, M. (2007). Guía clínica de alcoholismo. Barcelona, España: Socidrogalcohol.

Hernández, R., Fernández, C. y Baptista, P. (2014). Metodología de la investigación (6ta Ed.). México, D.F.: McGraw Gill.

Huillca, F. (13 de setiembre del 2008). Alcoholismo. La República, p. 22.

Instituto Nacional de Estadística e Informática. (2015). Enfermedades no transmisibles y transmisibles. Lima, Perú.

Laak, J. (1996). Las cinco grandes dimensiones de la personalidad. Perú. Revista de Psicología de la Pontificia Universidad Católica del Perú, Vol. XIV, núm. 2, p. 121-181.

Larsen, R. y Buss, D. (2005). Psicología de la personalidad. (2da ed.). México: McGraw Hill.

Leiva, E. (2009). Descripción de la Problemática del Alcoholismo en los Usuarios de las Cantinas de la Zona Sur del Barrio San Sebastián - Provincia y Departamento de Cajamarca durante el mes de octubre del 2009. (Proyecto de Investigación en Enfermería). Universidad Nacional de Cajamarca. Perú.

Mamani, A. y Silva, J. (2012). Consumo de alcohol y conductas sexuales de riesgo en estudiantes de secundaria de la Institución Educativa Nacional $N^{o} 72$ San Martín de Porres UGEL $N^{o} 03$ Magdalena del Mar. (Tesis para optar al título de Licenciado en Obstetricia). Universidad Nacional Mayor de San Marcos. Perú.

Mariátegui, J. (1984). Problemática del alcoholismo en el Perú. Una visión en perspectiva. Perú. Revista de Neuropsiquiatría. Vol. 47, p. 1-21.

Martínez, P. y Cassaretto, M. (2011) Validación del Inventario de los Cinco Factores NEO-FFI en español en estudiantes universitarios peruanos. D.F. México. Revista mexicana de Psicología. Vol. 28, núm. 1, p. 63-74.

Mestre, H., Viñas, M., Dutil, V. y Moya, J. (2005). Personalidad y consumo de alcohol en adolescentes. Madrid, España. Jornada de Fomento de la Investigadores. Universidad Jaume I.

Ministerio de Salud. (2004). Lineamiento para la acción en salud mental. Lima, Perú: Consejo Nacional de Salud. 
Moro, R. (18 de mayo del 2017). Argentina, el país con mayor consumo de alcohol en América Latina. Diario Infobae. Recuperado de: https://www.infobae.com

Organización Mundial de la Salud (1992). Clasificación de los trastornos mentales y del comportamiento CIE-10. Madrid, España: Editorial Médica Panamericana.

Organización Panamericana de la Salud (2008). Alcohol y atención primaria de la salud: Informaciones clínicas básicas para la identificación y manejo de riesgos y problemas. Washington D.C.

Paucar, W. (2010). Salud, Medio Ambiente y Agricultura. Salud en el Perú. Cuadernos de investigación universitaria. $\mathrm{N}^{\circ} 2$. Cusco, Perú. Universidad Nacional de San Antonio Abad del Cusco. p. 09-14,

Pilatti, A., Urrizaga, A., Chincolla, A. y Cupani, M. (2014). Facetas de personalidad relacionadas con el consumo elevado de alcohol en estudiantes universitarios de la provincia de Córdoba. Córdova, Argentina. Perspectivas en psicología. Vol. 11, p. 42-52. Universidad Nacional de Córdoba.

Ramírez, M. y Olivera, R. (2003). Características del consumo de alcohol en el distrito de Ccapacmarca. Cusco, Perú. SITUA - Revista Semestral de la Facultad de Medicina Humana. Universidad Nacional de San Antonio Abad del Cusco.

Richaud, M. (2002). Una revisión crítica del enfoque Lexicográfico y del modelo de los Cinco Factores. Lima Perú. Revista de Psicología de la Pontificia Universidad Católica del Perú. Vol. XX, núm. 1, p. 05-25.

Schmidt, V., Firpo, L., Vion, D., De Costa, M., Casella, L., Cuenya, L., Blum, G. y Pedrón, V. (2010). Modelo Psicobiológico de personalidad de Eysenck: una historia proyectada hacia el futuro. Buenos Aires, Argentina. Revista Internacional de Psicología. Vol. 11, núm. 02, p. 01-21. ISSN 1818-1023.

Schultz, D. y Schultz, S. (2010). Teoría de la personalidad. (9na ed.). México, D.F.: Cengage Learning.

Tello, S. (2010). El consumo de alcohol en estudiantes universitarios, las expectativas respecto a su uso y la autoeficacia de resistencia. (Tesis para optar el título de Licenciado en Psicología Clínica). Pontificia Universidad Católica del Perú. Perú.

Wayne, D. (2002). Bioestadísticas base para el análisis de las ciencias de la salud (4ta ed.). México, D.F.: Limusa S.A. 